\section{OP0175 CHARACTERIZATION OF NOVEL STROMAL-DERIVED AUTOANTIGENS RECOGNIZED BY RA SYNOVIAL MONOCLONAL ANTIBODIES}

E. Corsiero, L. Jagemann, E. Prediletto, C. Pitzalis, M. Bombardieri. Centre for Experimental Medicine \& Rheumatology, William Harvey Research Institute, London, United Kingdom

Background: We previously showed that up to $40 \%$ of RA synovial recombinant monoclonal antibodies (RA-rmAbs) generated from germinal center-like structure (GC-LS+) RA synovium recognize citrullinated antigens contained in neutrophils extracellular traps (NET) (1). The cellular source of other potential autoantigens targeted by the majority of locally differentiated $B$ cells remains undefined. Recently, RA-fibroblast-like synoviocytes (RA-FLS) have been implicated in the release of citrullinated antigens $(2,3)$. However, whether these cells are targeted by RA-rmAbs is still unknown.

Objectives: Here, we aimed to define the RA-rmAbs immunoreactivity towards i) RA-FLS and ii) identify potential stromal-derived autoantigens.

Methods: 67 RA-rmAbs were generated from single CD19+ B cells FACSsorted from fresh synovial cell suspensions following $\lg _{H}+V_{L}$ genes cloning (1). RA-rmAbs were tested by means of i) cell-based immunofluorescence assays with FLS of RA patients and controls (osteoarthritis (OA)-FLS and RA-dermal fibroblast (RA-DF)), ii) co-localization with stromal specific markers and iii) immunoenzymatic tests with co-localizing antigens. Control rmAbs were also used (Sjögren's syndrome/healthy donor-lgG rmAbs).

Results: Immunofluorescence on RA-FLS demonstrated reactivity of $21 \%$ of RA-rmAbs (14/67 rmAbs) towards cytoplasmic components of FLS. Only 4 rmAbs out of 14 were binding both FLS and NET components. For some rmAbs this reactivity was not specific to RA-FLS since it was also observed for OA-FLS and RA-DF. Interestingly, strong co-localization was observed with calreticulin (CRT) which in its citrullinated (cit-CRT) form has been previously shown to recognize the RA "shared epitope" HLA domain sequence (3). When tested in ELISA for native vs cit-CRT, $57 \%(8 / 14$ rmAbs) of the FLS-reactive RA clones showed binding to CRT with 4 out of 8 rmAbs displaying increased immunoreactivity towards cit-CRT. Controls rmAbs showed no reactivity to either FLS or CRT. Preliminary data suggest that RA patient serum preferentially recognize the lectin-like $\mathrm{N}$-terminal domain of CRT (4)

Conclusions: Here, we provide novel evidence that a subset of locally differentiated $B$ cells within RA synovial GC-LS can react towards RA-FLS derived antigens. Preliminary data suggest that part of this reactivity is directed towards CRT. Identification of immunodominant epitopes within CRT is under investigations.

References:

[1] Corsiero et al, ARD 2015.

[2] Sorice et al, Rheumatology 2016.

[3] Ling et al, AR 2013.

[4] Hong et al, JI 2010.

Disclosure of Interest: None declared

DOI: 10.1136/annrheumdis-2017-eular.3870

\section{OP0176 THE PARACASPASE MALT1 PLAYS A CENTRAL ROLE IN THE} PATHOGENESIS OF RHEUMATOID ARTHRITIS

E. Gilis ${ }^{1,2}$, J. Staal ${ }^{3,4}$, R. Beyaert ${ }^{4,5}$, D. Elewaut ${ }^{1,6}$. ${ }^{1}$ Department of Internal Medicine, Ghent University; ${ }^{2}$ Molecular Immunology and Inflammation Unit, VIB Inflammation Research Center; ${ }^{3}$ Department of Biomedical Molecular Biology, Ghent University; ${ }^{4}$ Molecular Signal Transduction in Inflammation; ${ }^{5}$ Department of Biomedical Molecular Biology; ${ }^{6}$ Molecular Immunology and Inflammation Unit, VIB Inflamamtion Research Center, Ghent, Belgium

Background: One of the hallmarks of many inflammatory arthritides is their strong linkage with $\mathrm{MHC}$-signalling, which is mirrored by the marked role for adaptive immunity. Accordingly, rheumatoid arthritis (RA) is characterized by the activation of auto-reactive T-cells and the development of auto-antibodies. T-cells may additionally respond to non-TCR mediated signals, which are essential in driving their effector functions. Pathways leading to the modulation of both innate and adaptive signals are therefore of marked interest to study in arthritic diseases. Objectives: The paracaspase MALT1 is a key player in the activation and proliferation of immune and non-immune cells. These cells include the lymphoid, myeloid and mast cells, indicating MALT1's crucial role in both innate and adaptive signaling (1). Therefore, MALT1 is regarded a promising target for the treatment of autoimmune diseases and defining its role in the pathogenesis of inflammatory arthritis is a critical first step.

Methods: To unravel MALT1's role in inflammatory arthritis, we initially assessed MALT1-activation in mice that were challenged with collagen-induced arthritis $(\mathrm{CIA})$, the prototype model for antigen-induced RA. We then addressed the role of MALT1 in the pathogenesis of inflammatory arthritis by challenging MALT1-deficient mice to distinct models of arthritis (CIA and CAIA) or by backcrossing MALT1-deficient mice to TNF ${ }^{\mathrm{DARE}}$ mice, representing an SpA-like model. Additionally, CIA was induced in CD4-specific MALT1-deficient mice to determine the importance of MALT1 in T-cells.

Results: We provide evidence that MALT1 plays a crucial role in the pathogenesis of RA as MALT1-deficent mice were completely protected against CIA. This complete protection was additionally observed in CD4-specific MALT1-deficient mice, indicating that the selective ablation of MALT1 in CD4-positive cells is sufficient for the observed resistance against CIA. CAIA on the other hand, which is a T- and B-cell independent model of RA, did not depend on the presence of MALT1, since both MALT1 $1^{+/+}$and MALT1 $1^{-/-}$mice showed comparable symptoms of RA. Interestingly, TNF ${ }^{\text {DARE }}$ mice that were deficient for MALT1 also showed a reduced enthesitis and ileitis phenotype, although TNF-concentration in the serum of these mice was higher compared to MALT $1^{+/+} \times$TNF $^{\text {DARE }}$ mice.

Conclusions: Overall, our data highlight that MALT1 plays a crucial role in the pathogenesis of inflammatory arthritis and represents an interesting candidate to target therapeutically.

References:

[1] Thome M. Multifunctional roles for MALT1 in T-cell activation. Nat Rev Immunol 2008; 8 (7): 495-500.

Acknowledgements: We thank Chris Vercruysse (Department of Basic Medical Sciences, University of Ghent, Belgium) for exerting the three-point-bending tests of the femurs and the lab of Prof. Dr. Luc Van Hoorebeke (Department of Physics and Astronomy) for the use of the $\mu \mathrm{CT}$-scanner.

Disclosure of Interest: None declared

DOI: 10.1136/annrheumdis-2017-eular.4924

\section{OP0177 N-GLYCOSYLATION SITES IN THE VARIABLE DOMAIN OF B CELL RECEPTORS SPECIFIC FOR CITRULLINATED ANTIGENS}

L.M. Slot ${ }^{1}$, R.D. Vergroesen ${ }^{1}$, L. Hafkenscheid ${ }^{1}$, M.T. Koning ${ }^{2}$, T. Rispens ${ }^{3}$ H. Veelken ${ }^{2}$, R.E. Toes ${ }^{1}$, H.U. Scherer ${ }^{1}$. ${ }^{1}$ Department of Rheumatology; ${ }^{2}$ Department of Hematology, Leiden University Medical Center, Leiden; ${ }^{3}$ Department of Immunopathology, Sanquin Research, Amsterdam, Netherlands

Background: Recent structural analysis of anti citrullinated protein antibodies (ACPA) in serum and synovial fluid of patients with rheumatoid arthritis (RA) revealed that the vast majority $(>90 \%)$ of secreted ACPA IgG molecules carry $\mathrm{N}$-glycans in the variable (Fab) domain. This remarkable degree of Fabglycosylation is absent from ACPA-depleted control IgG and from autoantibodies in other diseases. So far, it is unclear why ACPA carry this feature and which biological effects these glycans mediate in the context of RA. Of note, however, $\mathrm{N}$-glycosylation requires a specific amino acid consensus sequence in the protein backbone, which is very rare in germline-encoded variable region genes.

Objectives: To study the $B$ cell receptor (BCR) repertoire of ACPA-expressing $B$ cells to determine the frequency, origin and localisation of $\mathrm{N}$-glycosylation sites in ACPA Fab domains.

Methods: Citrullinated antigen-specific and non citrulline-reactive control B cells were identified in peripheral blood of ACPA-positive RA patients by antigenspecific tetramer staining and isolated by fluorescence activated cell sorting Cells were either sorted in pools and directly lysed, or sorted as single cells and cultured for two weeks followed by the detection of ACPA-positive culture wells by ELISA. Full-length immunoglobulin (Ig) rearrangements were identified by anchoring reverse transcription of Ig sequences, amplification by nested PCR and either next generation sequencing (NGS, PacBio platform) or, for single cell transcripts, Sanger sequencing (scSeq). Sequence reads were analysed using IMGT V-QUEST tools.

Results: The mean number of nucleotide mutations in heavy chains $(\mathrm{HC})$ of IgG BCRs derived from ACPA-expressing B cells was high (33 in NGS, 48 in scSeq samples; similarity to germline: $88 \%$ in NGS, $84 \%$ in scSeq). NGS identified 12 unique IgG clones derived from 4 donors, of which $10(83 \%)$ had at least one $\mathrm{N}$-glycosylation site in the $\mathrm{HC}$ or light chain (LC). scSeq identified 86 unique IgG clones derived from 6 donors, of which 68 (79\%) had $\mathrm{N}$-glycosylation sites. For $57 / 86 \mathrm{lgG}$ clones, we could determine the combination of HC and LC sequences In these, only $7(12 \%)$ clones had no sites, while $19(33 \%)$ had one, $23(41 \%)$ had two, $5(9 \%)$ had three and $3(4 \%)$ clones had four sites. 57 sites were found in the $\mathrm{HC}$ and 34 in the LC. All N-glycosylation sites were created by somatic mutations and not encoded in the germline sequence. Several sites were located in antigen-engaging regions. No correlation was found between the number of $\mathrm{N}$-glycosylation sites and the number of somatic mutations.

Conclusions: We demonstrate that B cell surface-expressed ACPA-IgG molecules carry a remarkably high frequency of $\mathrm{N}$-glycosylation sites in the Fab domain, all of which are generated by somatic mutation. This could indicate that ACPA-expressing $B$ cells acquire a selective survival advantage by introducing $\mathrm{N}$-glycosylation consensus sequences in the Fab domain, a process that is likely to occur under the influence of $T$ cell help and that could facilitate the break of tolerance to citrullinated antigens.

Disclosure of Interest: None declared

DOI: 10.1136/annrheumdis-2017-eular.5904

\section{OP0178 FIBROBLAST PRIMING IS COMMON TO MANY SITES, AND PSORIATIC SKIN FIBROBLASTS MAY ACQUIRE INFLAMMATORY MEMORY}

T. Crowley $^{1}$, J.D. O'Neil ${ }^{1}$, H.R. Adams ${ }^{1}$, T.W. Kragstrup ${ }^{2}$, A. Filer ${ }^{1}$,

C.D. Buckley ${ }^{1}$, A.R. Clark ${ }^{1} .{ }^{1}$ Institute of Inflammation and Ageing, University of Birmingham, Birmingham, United Kingdom; ${ }^{2}$ Biomedicine, Health, Aarhus University, Aarhus, Denmark

Background: Rheumatoid arthritis (RA) is a common chronic inflammatory 